\title{
Planar and Poly-arc Lombardi Drawings
}

\author{
Christian A. Duncan ${ }^{1}$, David Eppstein ${ }^{2}$, Michael T. Goodrich ${ }^{2}$, \\ Stephen G. Kobourov ${ }^{3}$, and Maarten Löffler ${ }^{2}$ \\ 1 Department of Computer Science, Louisiana Tech Univ., Ruston, Louisiana, USA \\ 2 Department of Computer Science, University of California, Irvine, California, USA \\ 3 Department of Computer Science, University of Arizona, Tucson, Arizona, USA
}

\begin{abstract}
In Lombardi drawings of graphs, edges are represented as circular arcs, and the edges incident on vertices have perfect angular resolution. However, not every graph has a Lombardi drawing, and not every planar graph has a planar Lombardi drawing. We introduce $k$-Lombardi drawings, in which each edge may be drawn with $k$ circular arcs, noting that every graph has a smooth 2-Lombardi drawing. We show that every planar graph has a smooth planar 3-Lombardi drawing and further investigate topics connecting planarity and Lombardi drawings.
\end{abstract}

\section{Introduction}

Motivated by the work of the American abstract artist Mark Lombardi [21], who specialized in drawings that illustrate financial and political networks, Duncan et al. [9]10] proposed a graph visualization called Lombardi drawings. These types of drawings attempt to capture some of the visual aesthetics used by Mark Lombardi, including his use of circular-arc edges and well-distributed edges around each vertex.

A vertex with circular arc edges extending from it has perfect angular resolution if the angles between consecutive edges, as measured by the tangents to the circular arcs at the vertex, all have the same degree. A Lombardi drawing of a graph $G=(V, E)$ is a drawing of a graph where every vertex is represented as a point, the edges incident on each vertex have perfect angular resolution, and every edge is represented as a line segment or circular arc between the points associated with adjacent vertices.

One drawback of previous work on Lombardi drawings is that (as we prove here) not every graph has a Lombardi drawing. In this paper we attempt to remedy this by considering drawings in which edges are represented by multiple circular arcs. This added generality allows us to draw any graph.

$k$-Lombardi Drawings. We define a $k$-Lombardi drawing to be a drawing with at most $k$ circular arcs per edge, with a 1-Lombardi drawing being equivalent to the earlier definition of a Lombardi drawing. We say that a $k$-Lombardi drawing is smooth if every edge is continuously differentiable, i.e., no edge in the drawing has a sharp bend. If a $k$-Lombardi drawing is not smooth, we say it is pointed. Fortunately, we do not need large values of $k$ to be able to draw all graphs: as we show, every graph has a smooth 2-Lombardi drawing. Interestingly, this result is hinted at in the work of Lombardi himself-Figure 1 shows a portion of a drawing by Lombardi that uses smooth edges consisting of two near-circular arcs. 


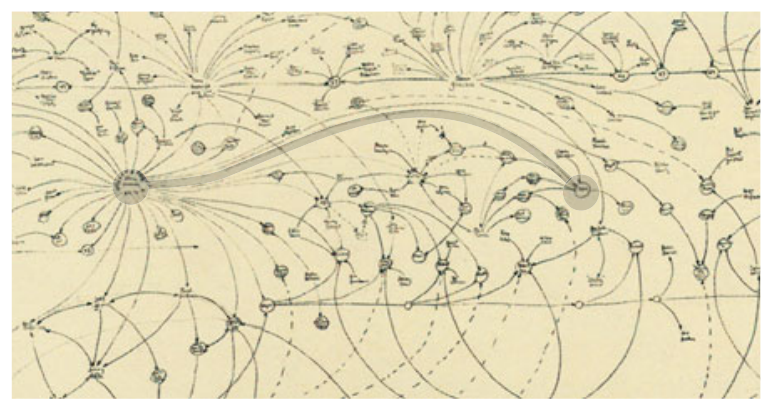

Fig. 1. A portion of Mark Lombardi, Chicago Outfit and Satellite Regimes, ca. 1931-83, 1998, $48.125 \times 96.6225$ inches (cat. no. 11) [21]. Note the highlighted smooth two-arc edge.

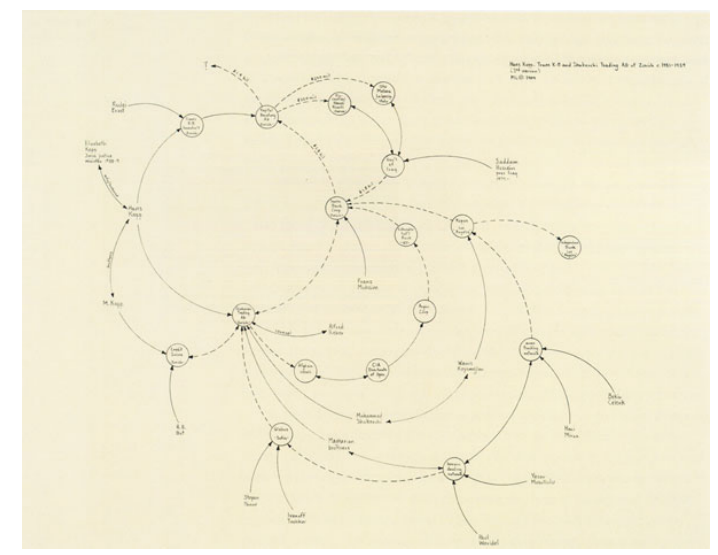

Fig. 2. Mark Lombardi, Hans Kopp, Trans K-B and Shakarchi Trading AG of Zurich, ca. 1981-89 (3rd Version), 1999, $20.25 \times 30.75$ inches (cat. no. 22) [21]

Planar Lombardi Drawings. Drawing planar graphs without crossings is a natural goal for graph drawing algorithms and is easily achieved when angular resolution is ignored. Lombardi himself avoided crossings in many of his drawings, as shown in Fig. 2] In previous work on Lombardi drawings, Duncan et al. [10] showed that there exist embedded planar graphs that have Lombardi drawings but do not have planar Lombardi drawings. Here we continue this investigation of planar Lombardi drawings and extend it to planar $k$-Lombardi drawings.

New Results. In this paper we provide the following results: (1) We find examples of graphs that do not have a Lombardi drawing, regardless of the ordering of edges around each vertex, thus strengthening an example from [10] of graphs for which a specific edge ordering cannot be drawn. (2) We find examples of planar 3-trees with no planar Lombardi drawing, strengthening an example from [10] of a planar graph with treewidth greater than three that is not planar Lombardi. (3) We show how to construct a smooth 2-Lombardi drawing for any graph, a smooth planar 2-Lombardi drawing of 
planar graph with maximum degree three, and a pointed planar 2-Lombardi drawing or a smooth planar 3-Lombardi drawing of any planar graph.

Other Related Work. In addition to the earlier work on Lombardi drawings, there is considerable prior work on graph drawing with circular-arc or curvilinear edges for the sake of achieving good, but not necessarily perfect, angular resolution [416]. There is also significant work on confluent drawings [7] 11|12]18,19], which use curvilinear edges not to separate edges but rather to bundle similar edges together and avoid edge crossings. Brandes and Wagner [3] provide a force-directed algorithm for visualizing train schedules using Bézier curves for edges and fixed positions for vertices. Finkel and Tamassia [14] extend this work by giving a force-directed method for drawing graphs with curvilinear edges where vertex positions are not fixed. Aichholzer et al. [1] show, for a given embedded planar triangulation with fixed vertex positions, it is possible to find a circular-arc drawing that maximizes the minimum angular resolution by solving a linear program. In addition, Matsakis [23] describes a force-directed approach to producing Lombardi drawings, but without an implementation. Chernobelskiy et al. [5], on the other hand, describe two functional Lombardi force-directed schemes that are based on the use of either dummy vertices or tangent forces but may not always achieve perfect angular resolution. Thus, to the best of our knowledge, none of this other related work correctly results in drawings of graphs having perfect angular resolution and curvilinear edges.

Alternatively, some previous work achieves good angular resolution using straightline drawings [6 15|22] or piecewise-linear poly-arc drawings [13 1720]. Di Battista and Vismara [6] characterize straight-line drawings of planar graphs with a prescribed assignment of angles between consecutive edges incident on the same vertex.

\section{$2 k$-Lombardi Drawings}

\subsection{Non-Lombardi Graphs}

Before investigating $k$-Lombardi drawings, we first establish the need for using poly-arc edges to be able to draw any graph. Although Duncan et al. [10] show a graph, Fig. 3(a) for which no Lombardi drawing is possible while preserving the given ordering of edges around each vertex, as Fig. 3(b) shows, if the ordering is not fixed, it is possible to create a valid Lombardi drawing for the graph. In this section, we provide a graph that has no Lombardi drawing irrespective of the edge ordering.

There are some complications in proofs of non-Lombardi counterexamples that differ from counterexamples in straight-line planar drawings. For example, if graph $G$ is non-Lombardi, this does not imply that all graphs $H \supset G$ are non-Lombardi because the addition of edges changes the angular resolution and can therefore dramatically change the subsequent placement of vertices. In addition, since the edge ordering is not fixed by the input, we must argue that any ordering forces a conflict.

Additional complications concern the density and symmetry of any possible counterexample. A $k$-degenerate graph is a graph that can be reduced to the empty graph by iteratively removing vertices of degree at most $k$. The graph in Fig. 3 is 3-degenerate, 


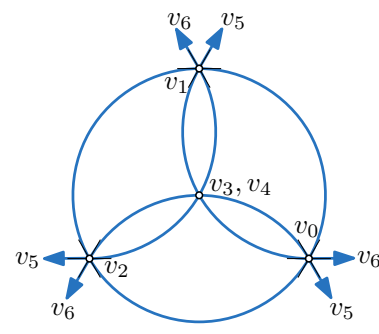

(a)

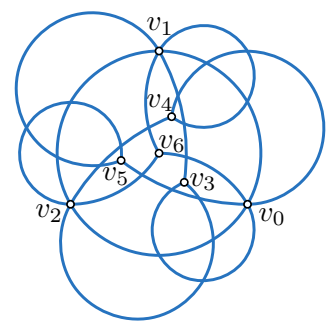

(b)

Fig. 3. A 7-vertex 3-degenerate graph that has no Lombardi drawing with the given edge ordering. (a) A Möbius transformation makes triangle $v_{0} v_{1} v_{2}$ equilateral, forcing both vertices $v_{3}$ and $v_{4}$ to be placed at the centroid and vertices $v_{5}$ and $v_{6}$ at the point at infinity; (b) A different ordering that does provide a Lombardi drawing.

and 3-degenerate graphs can be drawn Lombardi-style if we are willing to ignore vertexvertex and vertex-edge overlaps 1 Consequently, if a 3-degenerate graph is to be a counterexample, we must show that all vertex orderings force two vertices to overlap. Intuitively, 4-degenerate graphs should be more restrictive, but the simplest 4-degenerate graph, $K_{5}$, nevertheless has a circular Lombardi drawing. One reason is the fact that $K_{5}$ is extremely symmetrical. Therefore, we shall modify this graph to break its symmetry. We define our counterexample graph $G_{8}$ to be $K_{5}$ with the addition of three degree-one vertices causing one of the vertices of the original $K_{5}$ to have degree 5 and another to have degree 6, while the other three remain with degree 4; see Fig. 4(a)

Before we can establish our main theorem, we need to present a few geometric properties related to Lombardi drawings.

Property 1 ([10]). Let $A$ be a circular arc or line segment connecting two points $p$ and $q$ that both lie on circle $O$. Then $A$ makes the same angle to $O$ at $p$ that it makes at $q$. Moreover, for any $p$ and $q$ on $O$ and any angle $0 \leq \theta \leq \pi$, there exist either two arcs or a line segment and pair of collinear rays connecting $p$ and $q$, making angle $\theta$ with $O$, one lying inside and one outside of $O$.

We defer the proof of the next property, partially established in [10], to the full version of this paper [8].

Property 2. Suppose we are given two points $p=\left(p_{x}, p_{y}\right)$ and $q=\left(q_{x}, q_{y}\right)$ and associated angles $\theta_{p h}$ and $\theta_{q h}$ and an angle $\theta_{p q}$. Consider all pairs of circular arcs that leave $p$ and $q$ with angles $\theta_{p h}$ and $\theta_{q h}$ respectively (measured with respect to the positive horizontal axis) and meet at an angle $\theta_{p q}$. The locus of meeting points for these pairs of arcs is a circle. Moreover, the circle has radius $r_{c}=d_{p q} \csc \alpha / 2$ and center $\left(p_{x}+r_{c} \sin (\alpha+\beta), p_{y}-r_{c} \cos (\alpha+\beta)\right)$, where $\alpha=\left(\theta_{p h}-\theta_{q h}-\theta_{p q}\right) / 2, \beta$ is the angle formed by the ray from $p$ through $q$ with respect to the positive horizontal axis, and $d_{p q}$ is the distance between the points $p$ and $q$.

\footnotetext{
${ }^{1}$ Note that a drawing with vertex-vertex overlaps still must obey the perfect angular resolution constraints on the (possibly zero-length) edges, assuming such edge lengths are even allowed.
} 


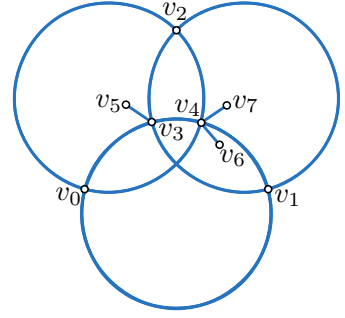

(a)

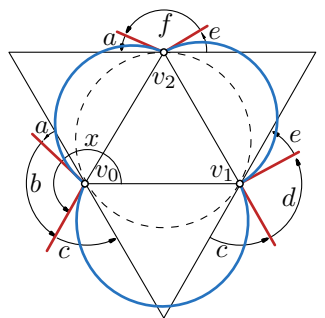

(b)

Fig. 4. (a) $G_{8}$ with the $K_{5}$ subgraph drawn Lombardi-style and additional edges shown. (b) Computing the twist for the three vertices 0,1 , and 2 . The twist for vertex 0 is $x$.

\section{Theorem 1. The graph $G_{8}$ is non-Lombardi.}

Proof. Let $v_{0}, v_{1}, v_{2}$ be the three vertices of $G_{8}$ with degree four. Let $v_{3}$ and $v_{4}$ be the vertices with degree five and six respectively. We do not care about the final placement of the degree-one vertices, whose main purpose is to alter the angular resolution of $v_{3}$ and $v_{4}$. Using a Möbius transformation we can assume that the first three vertices $v_{0}, v_{1}$, and $v_{2}$ are placed on the corners of a unit equilateral triangle such that $v_{0}$ and $v_{1}$ have positions $(0,0)$ and $(1,0)$ respectively. We shall show that for every edge ordering, the two vertices $v_{3}$ and $v_{4}$ cannot both be placed to maintain correctly their angular resolution and be connected to each other. We do this by establishing the algebraic equations for their positions based on the edge orderings of all vertices. We then show that such a set of equations has no solution for any valid assignment of orderings.

We first establish a notation for representing a specific edge ordering. For every vertex $v_{i}$ with neighbor $v_{j}$, let $k_{i j}$ represent the counterclockwise cyclic ordering of edge $\left(v_{i}, v_{j}\right)$ about $v_{i}$ with $k_{01}=0$ and $k_{i 0}=0$ for $i>0$. For example, in Fig. 4(a) the edge ordering around $v_{4}$ has $k_{41}=2, k_{42}=4, k_{43}=5, k_{46}=1$, and $k_{47}=3$. The twist $t_{i}$ of a vertex $v_{i}$ is the angle made by the arc extending from $v_{i}$ to the neighbor $v_{j}$ with $k_{i j}=0$. From the initial placement of $v_{0}, v_{1}$, and $v_{2}$ on an equilateral triangle and their respective edge orderings, we can uniquely determine the twists for each of these vertices; see Fig. 4(b). Since the three vertices lie on an equilateral triangle, the tangents to the circle defined by the three points also form an equilateral triangle. From Property 1 the angles formed by the arcs connecting each pair of vertices to the tangents at the circle yield matching (but undetermined) angles, labeled $a, c$, and $e$. The angles $b, d$, and $f$ are determined uniquely by the edge orderings as follows:

$$
b=2 \pi-k_{02} \pi / 2, \quad d=k_{12} \pi / 2, \quad f=2 \pi-k_{21} \pi / 2
$$

Noting that certain triplets of angles yield a value of $\pi$, we have the following three equations on three unknowns: $a+b+c=\pi+2 i_{0} \pi, c+d+e=\pi+2 i_{1} \pi$, and $e+f+a=$ $\pi+2 i_{2} \pi$. Solving for $a$ yields: $2 a=\pi-f-b+d+2\left(i_{0}-i_{1}+i_{2}\right) \pi$. For the twist for $v_{0}$, we wish to know the value of $x$, the angle for the arc from $v_{0}$ to $v_{1}$. Noting that $x=a+b+2 \pi / 3-2 i_{0} \pi$ and substituting in (1) yields $t_{0}=x=7 \pi / 6+\pi\left(k_{12}+k_{21}-\right.$ 
$\left.k_{02}\right) / 4+\left(i_{2}-i_{0}-i_{1}\right) \pi$. Noting that $t_{0}+c+\pi / 3=2 \pi$ yields $t_{1}=\pi-t_{0}$. Similarly, $t_{2}=\pi-a=5 \pi / 3-t_{0}-k_{02} \pi / 2+2 \pi\left(1-i_{0}\right)$.

The positions and orienting twists of the first three vertices also yield a unique position and twist for vertices $v_{3}$ and $v_{4}$. After determining these values, we shall show that in all orderings it is not possible to connect $v_{3}$ to $v_{4}$ with a single circular arc while still maintaining the proper angular resolution.

From Property 2] $v_{3}$ must lie on a circle $C_{01}$ defined by the neighbors $v_{0}$ and $v_{1}$ and their corresponding arc tangents. Similarly, it must lie on circles $C_{02}$ and $C_{12}$. The intersection of these three circles determines the position and orientation of $v_{3}$. Let us proceed to determine $C_{01}$. Letting $p=v_{0}$ and $q=v_{1}$, we have $\theta_{p h}=t_{0}+\pi k_{03} / 4$ and $\theta_{q h}=t_{1}+\pi k_{13} / 4$ and $\theta_{p q}=\pi\left(k_{31}-k_{30}\right) / 5=\pi k_{31} / 5$. From Property 2$]$ and the fact that $d_{p q}=1$, we can determine that $C_{01}$ has radius $r_{01}=\csc \alpha_{01} / 2$ and center $c_{01}=\left(r_{01} \sin \alpha_{01},-r_{01} \cos \alpha_{01}\right)=\left(1 / 2,-\cot \alpha_{01} / 2\right)$ with $\alpha_{01}=\left(\theta_{p h}-\theta_{q h}-\theta_{p q}\right) / 2=$ $t_{0}-\pi / 2+\pi\left(5 k_{03}-5 k_{13}-4 k_{31}\right) / 40$. Similarly, $C_{02}$ has radius $r_{02}=\csc \alpha_{02} / 2$ and center $c_{02}=\left(r_{02} \sin \left(\alpha_{02}+\pi / 3\right),-r_{02} \cos \left(\alpha_{02}+\pi / 3\right)\right)$ with $\alpha_{02}=t_{0}-5 \pi / 6+\pi\left(5 k_{03}+\right.$ $\left.10 k_{02}-5 k_{23}-4 k_{32}\right) / 40+\left(i_{0}-1\right) \pi$.

Given the circles and the position of $v_{0}$ at the origin, it is easy to determine the intersection of the two circles, one of which is $v_{0}$ and the other, if it even exists, must be $v_{3}$. Since $v_{0}$ must lie on the intersection, the line from $v_{0}$ to $v_{3}$ is perpendicular to the line, $\ell$, through the two centers. Moreover, $v_{3}$ is the reflection of $p$ about $\ell$. Thus, letting $v=\left(v_{x}, v_{y}\right)=c_{02}-c_{01}, c=v_{0}-c_{01}=-c_{01}$, and $v^{\perp}=\left(-v_{y}, v_{x}\right)$ yields $v_{3}=\frac{-2 c \cdot v^{\perp}}{v \cdot v} v^{\perp}$. To establish the twist $t_{3}$ at $v_{3}$ we observe from Property 1 that the angle $\alpha$ formed by the line $\ell_{03}$ from $v_{0}$ to $v_{3}$ and the tangent of the curve from $v_{0}$ to $v_{3}$ is the same as the tangent of the curve from $v_{3}$ to $v_{0}$ and the line $\ell_{03}$. Moreover, $\theta_{03}=t_{0}+k_{03} \pi / 4=\alpha+\beta_{03}$ and $t_{3}=\theta_{30}=\pi-\alpha+\beta_{03}$ where $\beta_{03}=\arctan \left(v_{3}(y) / \nu_{3}(x)\right)$ is the slope of $\ell_{03}$. From this, we can deduce that $t_{3}=\pi-t_{0}-k_{03} \pi / 4+2 \beta_{03}$. The exact same calculations can be used to compute $v_{4}$ and $t_{4}$.

As with the twists for $t_{3}$ and $t_{4}$, we can use Property 1 to determine the angles formed by the arc from $v_{3}$ to $v_{4}$ given their positions and twists. We know that the angles of the tangents to the arc at $v_{3}$ and $v_{4}$ are $\theta_{34}=t_{3}+k_{34} \pi / 5$ and $\theta_{43}=t_{4}+k_{43} \pi / 6$ respectively. Letting $\beta_{34}=\arctan \left(\left(v_{4}(y)-v_{3}(y)\right) /\left(v_{4}(x)-v_{3}(x)\right)\right)$ be the slope of the line from $v_{3}$ to $v_{4}$, we have that $\theta_{34}-\beta_{34}=\alpha$ and $\pi-\alpha=\theta_{43}-\beta_{34}$. Consequently, we have

$$
\theta_{34}+\theta_{43}=\pi+2 \beta_{34}
$$

Each specific edge ordering therefore yields a unique set of positions and twists for $v_{3}$ and $v_{4}$ as outlined above. To show that no Lombardi drawing is possible one must simply show that (2) does not hold for any edge ordering. Though there are a finite number of possible orderings and though symmetries could be used to reduce that number, the individual case analysis for such a proof appears to be quite unwieldy. Instead, we simply iterate over every possible edge ordering, applying these equations to a numerical algorithm that searches for a valid non-contradictory assignment. The full version of this paper [8] contains the Python code for this program. By running this program, one can see that no valid assignments are possible, concluding our proof. 


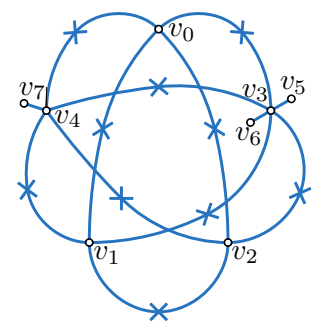

(a)

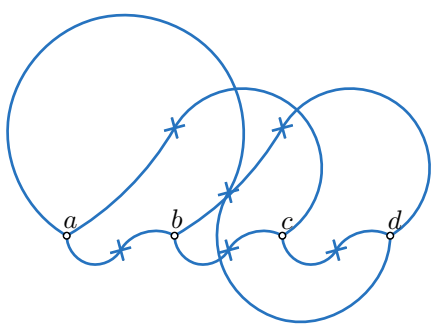

(b)

Fig. 5. (a) An example 2-Lombardi drawing of $G_{8}$. The bend points (not all of which are necessary) are shown with crossed marks. (b) An example 2-Lombardi drawing of $K_{4}$ with the vertices placed on a line and tangents oriented to force numerous inflection points.

Observing that we can take any Lombardi graph, subdivide an edge, and split the resulting new vertex into two degree-one vertices to produce a new Lombardi graph, we can get the following corollary, whose complete proof is provided in [8].

Corollary 1. There are an infinite amount of connected non-Lombardi graphs.

\subsection{Smooth 2-Lombardi Drawings}

If we want to draw Lombardi-style drawings for any given graph we have to relax one of the two requirements that specify Lombardi drawings. Here, we would like to avoid relaxing the requirement that edges have perfect angular resolution. Fortunately, we can achieve a Lombardi methodology for drawing any graph if we allow two circular arcs per edge; for example, see Fig. 5(a).

Since every 2-degenerate graph has a Lombardi drawing [10, Thm. 3] and since subdividing every edge in a graph results in a 2-degenerate graph, we readily obtain the following corollary, whose complete proof is found in the full version of the paper [8].

Corollary 2. Every graph has a smooth 2-Lombardi drawing. Furthermore, the vertices can be chosen to be in any fixed position.

As Fig. 5(b) illustrates, although we can place the vertices in any position with any initial orientation, an arc's smooth bend point might be an inflection point.

\section{Planar $k$-Lombardi Drawings}

\subsection{A Planar 3-Tree with No Planar Lombardi Drawing}

It is known that planar graphs do not necessarily have planar (non-crossing) Lombardi drawings. For example, Duncan et al. [10] show that the nested triangles graph must have edge crossings whenever there are 4 or more levels of nesting. While this graph 

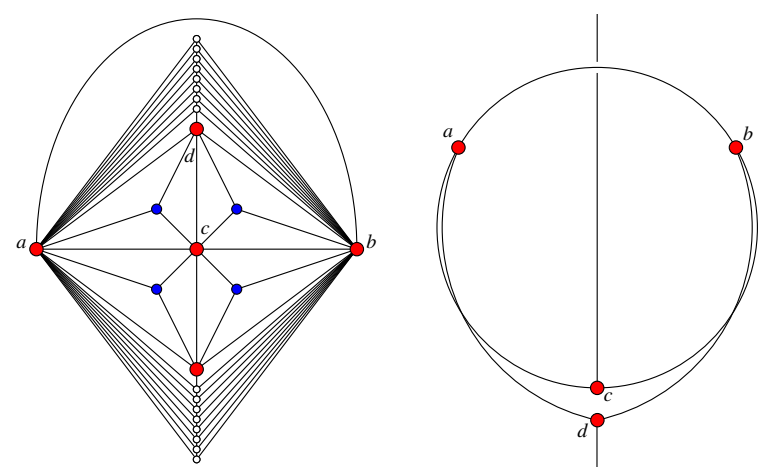

Fig. 6. Left: A planar 3-tree that has no planar Lombardi drawing. Right: For the $K_{4}$ subgraph defined by the four vertices $a, b, c$, and $d$, a drawing with the correct angles at each vertex will necessarily have crossings.

is 4-degenerate, even more constrained classes of planar graphs have no planar Lombardi drawings. Specifically, we can show that there exists a planar 3-tree that has no planar Lombardi realization. The planar 3-trees, also known as Apollonian networks, are the planar graphs that can be formed, starting from a triangle, by repeatedly adding a vertex within a triangular face, connected to the three triangle vertices, subdividing the face into three smaller triangles. These graphs have attracted much attention within the physics research community both as models of porous media with heterogeneous particle sizes and as models of social networks [2]. In addition, 3-trees are relevant for Lombardi drawings because they are examples of 3-degenerate graphs, which have nonplanar Lombardi drawings if vertex-vertex and vertex-edge overlaps are allowed.

\section{Theorem 2. There exists a planar 3-tree that has no planar Lombardi drawing.}

Proof. An example of a planar 3-tree that has no planar Lombardi drawing is given in Fig. 6, in the figure, sixteen small white vertices are shown, but our construction requires a sufficient number (which we do not specify precisely) in order to force the angle between arcs $a d$ and $a b$ to be arbitrarily close to $\pi$. The numbers of white vertices on the top and bottom of the figure should be equal. Because of this equality, the three arcs $a b, b c$, and $c a$ split the graph into two isomorphic subgraphs, and due to this symmetry they must each meet at angle $\pi$, necessarily forming a circle in any Lombardi drawing. By performing a Möbius transformation on the drawing, we may assume without loss of generality that these three points form the vertices of an equilateral triangle inscribed within the circle, as shown in the right of the figure. Then, according to our previous analysis of 3-degenerate Lombardi graph drawing, there is a unique point in the plane at which vertex $d$ may be located so that the arcs $a d, b d$, and $c d$ form the correct angle of $2 \pi / 3$ to each other and the correct angles to the three previous arcs $a b, b c$, and $c a$. However, as shown on the right of the figure, that unique point lies outside circle $a b c$ and causes multiple edge crossings in the drawing. 


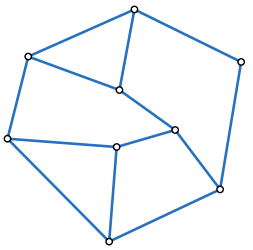

(a)

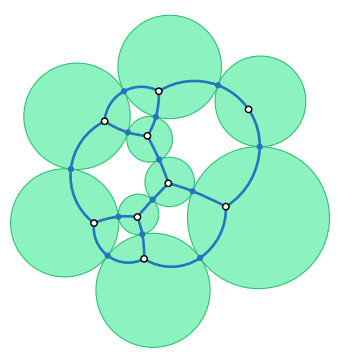

(b)

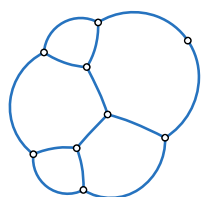

(c)

Fig. 7. (a) A planar graph of maximum degree 3. (b) A representation of the graph as tangent circles according to the Koebe-Andreev-Thurston theorem, together with arcs connecting each vertex perpendicularly to the disk tangency points. Layout generated using Ken Stephenson's CirclePack software. (c) The final smooth 2-Lombardi drawing.

\subsection{Smooth Planar 2-Lombardi Drawings of Planar Max-Degree-3 Graphs}

Lemma 1. Given a circle $C$ and three points $a, b$, and $c$ on it, there exists a point $p$ inside $C$ such that we can draw three edges from $p$ to $a, b$, and $c$ as circular arcs that are all perpendicular to $C$ and meet inside $p$ at angle $2 \pi / 3$.

Proof. We can find a Möbius transform $\tau$ that maps the circle to itself, mapping $a, b$, and $c$ to three points $a^{\prime}, b^{\prime}$ and $c^{\prime}$ that are $2 \pi / 3$ radians apart on the circle. For these three points, the three edges can be drawn as radii of the circle meeting at the center point $p^{\prime}$. The inverse transformation to $\tau$ maps $p^{\prime}$ to $p$ and maps these three radii to circular arcs with the desired property.

Theorem 3. Every planar graph $G$ of maximum degree 3 has a smooth planar 2Lombardi drawing.

Proof. We apply the Koebe-Andreev-Thurston theorem to create a representation of $G$ as the intersection graph of tangent circles; see Fig. 7(b) Each circle has three contact points that will be the bend points of its incident edges. Applying Lemma1 to the circles yields a vertex and half-edge drawing inside each disk. At each contact point two halfedges meet at angle $\pi$, resulting in a smooth planar 2-Lombardi drawing of $G$.

\subsection{Pointed Planar 2-Lombardi Drawings of Planar Graphs}

We now show that every planar graph allows a planar 2-Lombardi drawing with pointed joints. The approach is similar to the previous section, but the drawing method inside the disks is different. We need the following lemmas, which are illustrated in Fig. 8 We defer the proof of the first lemma to the full version of the paper [8].

Lemma 2. Let $C$ be a circle and $P$ be a set of $n$ points on $C$. Additionally suppose that the four integers $n_{1}, n_{2}, n_{3}, n_{4}$ sum up to $n$ and satisfy the inequalities $\lfloor n / 4\rfloor \leq$ 


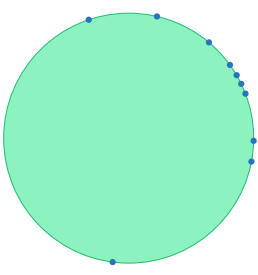

(a)

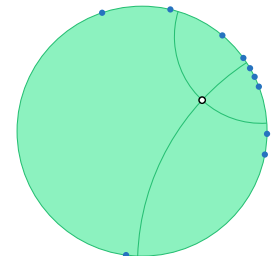

(b)

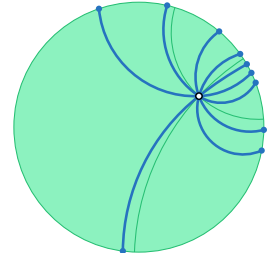

(c)

Fig. 8. (a) A disk with a set of connection points on its boundary. (b) A placement for the vertex in the disk that divides the connection points into four quadrants. (c) The actual connections are not fixed and guaranteed not to intersect.

$n_{i} \leq\lceil n / 4\rceil$ and $\lfloor n / 2\rfloor \leq n_{i}+n_{(i+1) \bmod 4} \leq\lceil n / 2\rceil$. Then there exist two circles $A$ and $B$ disjoint from $P$ such that $A, B$, and $C$ are pairwise perpendicular and such that $A$ and $B$ subdivide $P$ into four sets of cardinalities $n_{1}, n_{2}, n_{3}$ and $n_{4}$.

Lemma 3. Given a circle $C$ and a set $P$ of $n$ points on $C$, there exists a point $p$ in $C$ such that we can draw $n$ edges from $p$ to the points in $P$ as circular arcs that lie completely inside $C$, do not cross each other, and meet in $p$ at angle $2 \pi / n$.

Proof. Draw $n$ ports around a point with equal angles, and draw two perpendicular lines through the point (not coinciding with any ports), and count the number of points in each quadrant. Let these numbers be $n_{1}, \ldots, n_{4}$ and find two circles $A$ and $B$ as in Lemma2 Then we place $p$ at their intersection point inside $C$. Now orient the ports at $p$ such that each quadrant has the correct number of ports.

Within any quadrant, there is a circular arc tangent to $C$ at the point where it is crossed by $B$ and tangent to $A$ at point $p$; this can be seen by using a Möbius transformation to transform $A$ and $B$ into a pair of perpendicular lines, after which the desired arc has half the radius of $C$. By the intermediate value theorem, there are two circular arcs from $p$ to any point $q$ on the boundary arc of the quadrant that remain entirely within the quadrant and are tangent to $A$ and $B$ respectively. By a second application of the intermediate value theorem, there is a unique circular arc that connects $p$ to each connection point on the boundary of $C$ such that the outgoing direction at $p$ matches the port and such that the arc remains entirely within its quadrant.

Any two arcs lying in the same quadrant belong to two circles that cross at $p$ and at one more point. Whether that second crossing point is inside or outside of the quadrant can be determined by the relative ordering of the two arcs at $p$ and on the boundary of the quadrant. However, since the ordering of the ports and of the connection points is the same, none of the crossings of these circles are within $C$, so no two arcs cross.

Theorem 4. Every planar graph has a pointed planar 2-Lombardi drawing.

Proof. As before, we first obtain a touching-circles representation of graph $G$ using the Koebe-Andreev-Thurston theorem. Each vertex $v$ in $G$ is represented by a circle $C$; place $v$ together with arcs connecting it to the set of contact points on $C$ using Lemma 3 The arcs meet up at the contact points to form (non-smooth) 2-Lombardi edges. 


\subsection{Smooth Planar 3-Lombardi Drawings of Planar Graphs}

Although the 2-Lombardi planar realization above has non-smooth bends in each edge, as we now show, every planar graph also has a smooth planar 3-Lombardi drawing. It seems likely that one can obtain a smooth planar 3-Lombardi drawing from a planar graph $G$ by perturbing each edge of a straight-line drawing of $G$ into a curve formed by two very small circular arcs near each endpoint of the edge, connected to each other by a straight segment. However, the details of this construction are messy. An alternative construction is much simpler, once Theorem 4 is available.

Theorem 5. Every planar graph has a smooth planar 3-Lombardi drawing.

Proof. Find a pointed planar 2-Lombardi drawing by Theorem 4 For each pointed bend of the drawing formed by two circular $\operatorname{arcs} a_{1}$ and $a_{2}$, replace the bend by a third circular arc tangent to both $a_{1}$ and $a_{2}$, with the two points of tangency close enough to the bend to avoid crossing any other edge.

\section{Conclusions}

We have proven several new results about the planarity of Lombardi drawings and about the classes of graphs that can be drawn as $k$-Lombardi drawings rather than as Lombardi drawings. However, several problems remain open, including the following:

1. Characterize the subclasses of planar graphs having Lombardi planar realizations and those having smooth 2-Lombardi planar realizations.

2. Bound the (change in) curvature of edge segments in $k$-Lombardi drawings.

3. Address area and resolution requirements for Lombardi drawings of graphs.

Acknowledgments. This research was supported in part by the National Science Foundation under grants CCF-0830403, CCF-0545743, and CCF-1115971, by the Office of Naval Research under MURI grant N00014-08-1-1015, and by the Louisiana Board of Regents through PKSFI Grant LEQSF (2007-12)-ENH-PKSFI-PRS-03.

\section{References}

1. Aichholzer, O., Aigner, W., Aurenhammer, F., Dobiášová, K.Č., Jüttler, B.: Arc triangulations. In: Proc. 26th Eur. Worksh. Comp. Geometry (EuroCG 2010), Dortmund, Germany, pp. 17-20 (2010)

2. Andrade Jr., J.S., Herrmann, H.J., Andrade, R.F.S., da Silva, L.R.: Apollonian networks: Simultaneously scale-free, small world, Euclidean, space filling, and with matching graphs. Physics Review Letters 94, 018702 (2005); arXiv:cond-mat/0406295

3. Brandes, U., Wagner, D.: Using graph layout to visualize train interconnection data. J. Graph Algorithms Appl. 4(3), 135-155 (2000), http://jgaa.info/accepted/00/BrandesWagner00.4.3.pdf

4. Cheng, C.C., Duncan, C.A., Goodrich, M.T., Kobourov, S.G.: Drawing planar graphs with circular arcs. Discrete Comput. Geom. 25(3), 405 (2001), doi:10.1007/s004540010080 
5. Chernobelskiy, R., Cunningham, K., Goodrich, M.T., Kobourov, S.G., Trott, L.: Forcedirected Lombardi-style graph drawing. In: van Kreveld, M., Speckmann, B. (eds.) GD 2011. LNCS, vol. 7034, pp. 320-331. Springer, Heidelberg (2011)

6. Di Battista, G., Vismara, L.: Angles of planar triangular graphs. SIAM J. Discrete Math. 9(3), 349-359 (1996), doi:10.1137/S0895480194264010

7. Dickerson, M., Eppstein, D., Goodrich, M.T., Meng, J.Y.: Confluent drawings: Visualizing non-planar diagrams in a planar way. J. Graph Algorithms Appl. 9(1), 31-52 (2005), http://jgaa.info/accepted/2005/Dickerson+2005.9.1.pdf

8. Duncan, C.A., Eppstein, D., Goodrich, M.T., Kobourov, S.G., Löffler, M.: Planar and polyarc Lombardi drawings. ArXiv e-prints abs/1109.0345, arXiv:1109.0345 (September 2011)

9. Duncan, C.A., Eppstein, D., Goodrich, M.T., Kobourov, S.G., Nöllenburg, M.: Drawing Trees With Perfect Angular Resolution and Polynomial Area. In: Brandes, U., Cornelsen, S. (eds.) GD 2010. LNCS, vol. 6502, pp. 183-194. Springer, Heidelberg (2011), doi:10.1007/978-3-642-18469-7_17; arXiv:1009.0581

10. Duncan, C.A., Eppstein, D., Goodrich, M.T., Kobourov, S.G., Nöllenburg, M.: Lombardi Drawings of Graphs. In: Brandes, U., Cornelsen, S. (eds.) GD 2010. LNCS, vol. 6502, pp. 195-207. Springer, Heidelberg (2011), doi:10.1007/978-3-642-18469-7_18; arXiv: 1009.0579

11. Eppstein, D., Goodrich, M.T., Meng, J.Y.: Delta-Confluent Drawings. In: Healy, P., Nikolov, N.S. (eds.) GD 2005. LNCS, vol. 3843, pp. 165-176. Springer, Heidelberg (2006), doi:10.1007/11618058_16; arXiv:cs/0510024v1

12. Eppstein, D., Goodrich, M.T., Meng, J.Y.: Confluent layered drawings. Algorithmica 47(4), 439-452 (2007), doi:10.1007/s00453-006-0159-8

13. Eppstein, D., Löffler, M., Mumford, E., Nöllenburg, M.: Optimal 3D Angular Resolution for Low-Degree Graphs. In: Brandes, U., Cornelsen, S. (eds.) GD 2010. LNCS, vol. 6502, pp. 208-219. Springer, Heidelberg (2011), doi:10.1007/978-3-642-18469-7_19; arXiv: 1009.0045

14. Finkel, B., Tamassia, R.: Curvilinear Graph Drawing Using the Force-Directed Method. In: Pach, J. (ed.) GD 2004. LNCS, vol. 3383, pp. 448-453. Springer, Heidelberg (2005), doi:10.1007/978-3-540-31843-9_46

15. Garg, A., Tamassia, R.: Planar Drawings and Angular Resolution: Algorithms and Bounds. In: van Leeuwen, J. (ed.) ESA 1994. LNCS, vol. 855, pp. 12-23. Springer, Heidelberg (1994), doi:10.1007/BFb0049393

16. Goodrich, M.T., Wagner, C.G.: A framework for drawing planar graphs with curves and polylines. Journal of Algorithms 37(2), 399-421 (2000), doi:10.1006/jagm.2000.1115

17. Gutwenger, C., Mutzel, P.: Planar Polyline Drawings With Good Angular Resolution. In: Whitesides, S.H. (ed.) GD 1998. LNCS, vol. 1547, pp. 167-182. Springer, Heidelberg (1999), doi:10.1007/3-540-37623-2_13

18. Hirsch, M., Meijer, H., Rappaport, D.: Biclique Edge Cover Graphs and Confluent Drawings. In: Kaufmann, M., Wagner, D. (eds.) GD 2006. LNCS, vol. 4372, pp. 405-416. Springer, Heidelberg (2007), doi:10.1007/978-3-540-70904-6_39

19. Holten, D., van Wijk, J.J.: Force-directed edge bundling for graph visualization. Computer Graphics Forum 28, 983-990 (2009), doi:10.1111/j.1467-8659.2009.01450.x

20. Kant, G.: Drawing planar graphs using the canonical ordering. Algorithmica 16, 4-32 (1996), doi:10.1007/BF02086606

21. Lombardi, M., Hobbs, R.: Mark Lombardi: Global Networks. Independent Curators (2003)

22. Malitz, S., Papakostas, A.: On the angular resolution of planar graphs. SIAM J. Discrete Math. 7(2), 172-183 (1994), doi:10.1137/S0895480193242931

23. Matsakis, N.: Transforming a random graph drawing into a Lombardi drawing. ArXiv ePrints abs/1012.2202, arXiv:1012.2202 (December 2010) 